\title{
Norois
}

Environnement, aménagement, société

\section{La ville moyenne touristique est-elle encore une ville moyenne?}

Une approche par les populations présentes

Is the medium-sized tourist city still a medium-sized city? An approach based on present populations

\section{Luc Vacher et Didier Vye}

\section{OpenEdition}

\section{Journals}

Édition électronique

URL : https://journals.openedition.org/norois/4359

DOI : $10.4000 /$ norois.4359

ISBN : 978-2-7535-2634-1

ISSN : $1760-8546$

\section{Éditeur}

Presses universitaires de Rennes

Édition imprimée

Date de publication : 30 décembre 2012

Pagination : 75-91

ISBN : 978-2-7535-2633-4

ISSN : 0029-182X

\section{Référence électronique}

Luc Vacher et Didier Vye, «La ville moyenne touristique est-elle encore une ville moyenne? », Norois

[En ligne], 225 | 2012, mis en ligne le 30 décembre 2014, consulté le 14 janvier 2022. URL : http:// journals.openedition.org/norois/4359; DOI : https://doi.org/10.4000/norois.4359 


\title{
La ville moyenne touristique est-elle encore une ville moyenne? Une approche par les populations présentes
}

\author{
Is the Medium-sized Tourist City still a Medium-sized City? \\ An Approach Based on Present-in-area Population
}

\author{
Luc VACHER, Didier Vye
}

AGÎLE - UMR 7266 Littoral, Environnement, Sociétés LIENSs, (Université de La Rochelle) Institut du Littoral et de l'Environnement, 2, rue Olympe-de-Gouges-17000 LA RochelLE, France(lvacher@univ-lr.fr)(dvye@univ-lr.fr)

\begin{abstract}
Résumé : L'expression «ville moyenne » regroupe des catégories de villes très hétérogènes. L'objectif de cette communication est de mieux cerner un profil de ville particulier, la ville moyenne touristique située en bord de mer. À partir du cas de La Rochelle, il s'agit de montrer dans quelle mesure cette agglomération, bien que de taille moyenne (120000 habitants), présente toutefois des caractéristiques qui la surclasse dans la hiérarchie urbaine en raison notamment de la présence régulière ou saisonnière de populations non comptabilisées, de la diversité des populations qui la fréquentent ainsi que de l'ampleur de son attractivité touristique et résidentielle. La communication s'appuiera essentiellement sur les résultats de l'Observatoire des pratiques de tourisme et de loisir de l'UMR LIENSs de l'université de La Rochelle qui a recueilli sur les 5 plages principales de l'agglomération rochelaise plus de 5600 questionnaires d'enquêtes réalisées en saison estivale depuis 2008.
\end{abstract}

Abstract: The term "medium-sized city" encompasses a wide variety of city categories. The aim of this paper is to more clearly define a specific city profile, the medium-sized tourist city lying by the sea. With reference to the case of La Rochelle, we will show the extent to which this conurbation, although of medium size (120,000 inhabitants), nonetheless presents characteristics which push it up the urban rankings largely due to the regular or seasonal presence of unrecorded populations, the diversity of the populations found there and the scale of its attractiveness on both the tourism and residential levels. The paper will primarily draw on the findings of the monitoring unit for tourism and leisure practices of the UMR LIENSs at the University of La Rochelle which has collected over 5,600 survey questionnaires on the 5 main beaches of the La Rochelle conurbation in the summer season since 2008.

Mots clés : ville moyenne, tourisme, population présente, urbanité, attractivité

Keywords: medium-sized city, tourism, present population, urbanity, attractiveness

\section{INTRODUCTION}

En France, en Europe et dans le monde, l'expression «ville moyenne » regroupe des catégories de villes très disparates tant d'un point de vue du nombre d'habitants, des fonctions que de la configuration territoriale dans laquelle elles s'inscrivent. L'objectif de cette communication est de mieux cerner un profil de ville particulier, la ville moyenne touristique située en bord de mer. En s'appuyant sur 
le cas de La Rochelle, grande destination touristique du littoral français, il s'agit de montrer dans quelle mesure une ville touristique possède des caractéristiques qui la surclasse dans la hiérarchie urbaine.

En effet, comme d'autres villes touristiques atlantiques (Lorient, Saint-Malo, Biarritz...), La Rochelle est une ville de taille moyenne, et ce quels que soient les seuils statistiques, politiques ou les périmètres (commune-centre, agglomération, aire urbaine) de référence en France depuis 20 ans. Son agglomération, au sens de l'unité urbaine de l'INSEE, regroupe 119700 habitants en 2007, ce qui la positionne comme telle selon les critères démographiques utilisés en France. En effet, selon Frédéric Santamaria, une ville moyenne est une agglomération comprenant entre 20000 et 200000 habitants (Santamaria, 2000). Peuplée de 186200 personnes, son aire urbaine est également « moyenne " selon les critères de la DATAR selon laquelle la ville moyenne est une aire urbaine comprenant entre 30000 et 200000 habitants $^{1}$. Enfin, avec 76800 habitants, la commune-centre possède les critères requis pour intégrer la Fédérations des Maires des Villes Moyennes (commune-centre comprenant entre 20000 et 100000 habitants et/ ou possédant une préfecture) bien que la municipalité ait fait le choix de rejoindre l'Association des Maires de Grandes Villes de France qui regroupe les communes ou intercommunalités de plus de 100000 habitants.

Mais ce surclassement est-il seulement symbolique et politique? Considérant le fait qu'une ville touristique en général et une ville littorale en particulier attirent une population beaucoup plus nombreuse que la population résidante à l'année, la notion de ville moyenne appliquée à cette catégorie de ville semble alors à relativiser. Mais, derrière ce simple constat partagé par quiconque ayant déjà fréquenté une ville touristique en plein saison se pose une question simple : comment appréhender et quantifier cette population présente? Ce sera l'objet d'une première partie.

De plus, cette population supplémentaire fréquentant la ville touristique n'a pas uniquement pour conséquence une augmentation, même provisoire, de la taille démographique de la ville. La ville touristique regroupe aussi plusieurs types de populations qui ne

1. Source : Programme « 20 villes moyennes témoins » lancé en 2007. se résument pas aux seuls « touristes » et aux seuls résidents. Or, selon M. Lussault (2003) il existe un gradient d'urbanité fondée sur l'intensité du couple densité/diversité. Dès lors, un «supplément d'urbanité » liée à la diversité des populations qui la fréquente peut également contribuer à surclasser une ville moyenne. Au sein de la ville touristique littorale, la question de l'urbanité peut être analysée à partir des principaux lieux de fréquentation, en particulier les plages, qui sont également des lieux de coprésence c'est-à-dire, marqués par une présence simultanée de populations différenciées dans un même lieu. De ce fait, cette diversité des populations présentes ne tranche-t-elle pas avec une des représentations de la ville moyenne considérée par F. Taulelle (2010) comme « facilement appréhendable ${ }^{2}$ »? La deuxième partie tentera de répondre à cette interrogation en traitant le cas des cinq principales plages de l'agglomération rochelaise.

Enfin, la ville touristique est une ville attractive. Cette attractivité, touristique mais aussi résidentielle, dessine une aire d'influence de la ville qui dépasse largement ce que son poids démographique laisse suggérer. Cette attractivité est également marquée par une forte composante métropolitaine, essentiellement francilienne, dans le lieu d'origine des touristes comme dans celui des nouveaux résidents. L'ampleur et les caractéristiques de cette attractivité ne contribueraient-elles pas à instaurer une proximité entre la ville moyenne touristique et la métropole? Cela fera l'objet d'une troisième partie.

Cette étude s'appuiera essentiellement sur les résultats de l'Observatoire des pratiques de tourisme et de loisir ECOP de l'UMR LIENSs (Littoral, Environnement, Sociétés) de l'Université de La Rochelle ${ }^{3}$. Cet observatoire lancé en 2008 a déjà permis de recueillir auprès des habitants, des résidents secondaires, des touristes ou des excursionnistes, plus de 5600 questionnaires réalisés sur les plages de l'agglomération rochelaise durant la saison estivale.

\footnotetext{
2. «Ce sont des villes facilement appréhendables : on en a vite fait le tour, leur structure urbaine est facile à comprendre » (Taulelle, 2010, p. 153). F. Taulelle précise que même si ces images sont des représentations mentales, elles composent néanmoins un «modèle de ville ».

3. Dans le cadre de cet observatoire, les auteurs ont bénéficié du soutien du CPER 2007-2011 incluant des crédits FEDER.
} 


\section{LA VILLE TOURISTIQUE LITTORALE : QUAND LA POPULATION PRÉSENTE DÉPASSE LA POPULATION RÉSIDENTE}

\section{L'intérêt d'évaluer les populations présentes}

En France, la population de référence est celle issue des recensements de population réalisés par l'INSEE qui quantifie la population « résidente » (à l'année) dans chaque logement considéré comme résidence principale. Celle-ci détermine, entre autres, le nombre d'élus locaux, le budget communal (via la Dotation Générale de Fonctionnement), la nécessité ou non de maintenir ou de renforcer des services publics. Elle a également longtemps servi de référence dans les études et analyses en matière d'aménagement des territoires.

Or, face à l'explosion des mobilités, notamment de tourisme et de loisir, et à l'augmentation du phénomène de multirésidentialité, le calcul de cette population résidente ne permet plus de saisir la réalité démographique d'un lieu. C'est particulièrement vrai pour les territoires touristiques dont les pouvoirs publics locaux doivent composer avec une population particulièrement fluctuante afin d'adapter leur capacité d'accueil, de calibrer leurs équipements (stations d'épuration, places de stationnement...) ou d'évaluer l'impact environnemental de la fréquentation. De plus, l'apport supplémentaire de populations, par les besoins qu'elles nécessitent, contribue à la création de richesses économiques. C'est la notion d'économie présentielle qui à partir de 2003 s'est progressivement substituée à celle d'économie résidentielle qui ne traitait que des richesses induites par les résidents permanents ou secondaires (Davezies, 2009). Ainsi, depuis janvier 2010, l'INSEE sépare l'économie présentielle qui regroupe les « activités mises en œuvre localement pour la production de biens et de services visant la satisfaction des besoins de personnes présentes dans la zone, qu'elles soient résidentes ou touristes ${ }^{4} »$ de l'économie non présentielle, calculée par défaut. L'économie présentielle devient donc la clé de voûte de l'analyse économique territoriale. Toutefois, dans cette définition, il n'est pas fait mention des visiteurs à la journée.

\footnotetext{
4. Source : insee.fr, rubrique définitions et méthodes. URL : [http://www. insee. $\mathrm{fr} / \mathrm{fr} /$ methodes/default.asp? page=definitions/sphere.htm]. Consulté le 28 mars 2011 .
}

Ainsi, les études permettant de quantifier au mieux la fréquentation d'un lieu se sont multipliées ces dernières années avec l'émergence de la notion de population présente. Cette dernière désigne la population présente dans un lieu à un moment donné. Elle possède donc une double dimension, à la fois spatiale et temporelle (Terrier, 2009). La quantification de la population présente est un vrai défi scientifique et méthodologique. En France, le travail de référence reste celui engagé par Christophe Terrier pour le compte du ministère du Tourisme (Terrier, 2006). Il s'agit d'une estimation reposant entre autres sur l'enquête "Suivi de la demande touristique »(SDT) réalisée chaque mois afin de connaitre les déplacements touristiques d'un échantillon de 20000 personnes âgées de 15 ans et plus. Prenant comme base la population résidente à l'année, l'estimation est effectuée jour par jour à l'échelle d'une année. Elle est réalisée sur l'ensemble du territoire métropolitain français et tient compte aussi bien des mobilités à l'intérieur de l'hexagone grâce l'enquête SDT que de celles en provenance de l'étranger, ces dernières étant calculées à partir de plusieurs sources : enquêtes aux frontières, enquêtes mensuelles de fréquentation des hôtels et des campings.

\section{Évaluer les populations présentes dans une agglomération touristique littorale}

Cette étude ambitieuse ne permet cependant pas d'évaluer la population présente à l'échelle d'une ville moyenne comme La Rochelle, l'unité de base de la méthodologie de C. Terrier étant le département. Ainsi, les flux calculés en fonction des présents et des absents sont des flux interdépartementaux auxquels s'ajoutent les flux touristiques en provenance de l'étranger. Cette échelle de référence écarte ainsi les déplacements touristiques à l'intérieur d'un même département et ne permet pas de quantifier la population présente à l'échelle de l'espace urbain sauf pour le cas particulier des départements presqu'entièrement urbanisés et organisés autour d'une seule métropole (départements franciliens, Rhône...). L'approche de C. Terrier nous permet donc de savoir que la population moyenne présente sur l'année en Charente-Maritime était supérieure en 2005 de 16,3 \% à celle de la population résidente, soit 95800 habitants supplémen- 
taires, mais sans que l'on connaisse la proportion concentrée sur le littoral d'une part et dans les villes (La Rochelle) ou stations touristiques (Royan) d'autre part (figure 1).

La recherche d'une maille plus fine est une préoccupation importante dans l'étude des populations présentes. Ainsi, l'INSEE a lancé en 2009 une étude semblable à l'échelle des bassins de vie qui devrait bientôt voir le jour. Les territoires insulaires, pratiques à analyser car possédant en général un seul point d'entrée et de sortie, sont ainsi l'objet de nombreuses analyses de fréquentation fines sur le plan quantitatif comme sur le plan qualitatif. En France, citons la thèse de S. Le Berre (2008) sur l'archipel breton de Chausey réalisée dans le cadre de l'observatoire dirigé par le géographe Louis Brigand, les travaux de la DREAL Pays de la Loire sur l'Île d'Yeu en Vendée (Potier et al., 2009), ou dif-
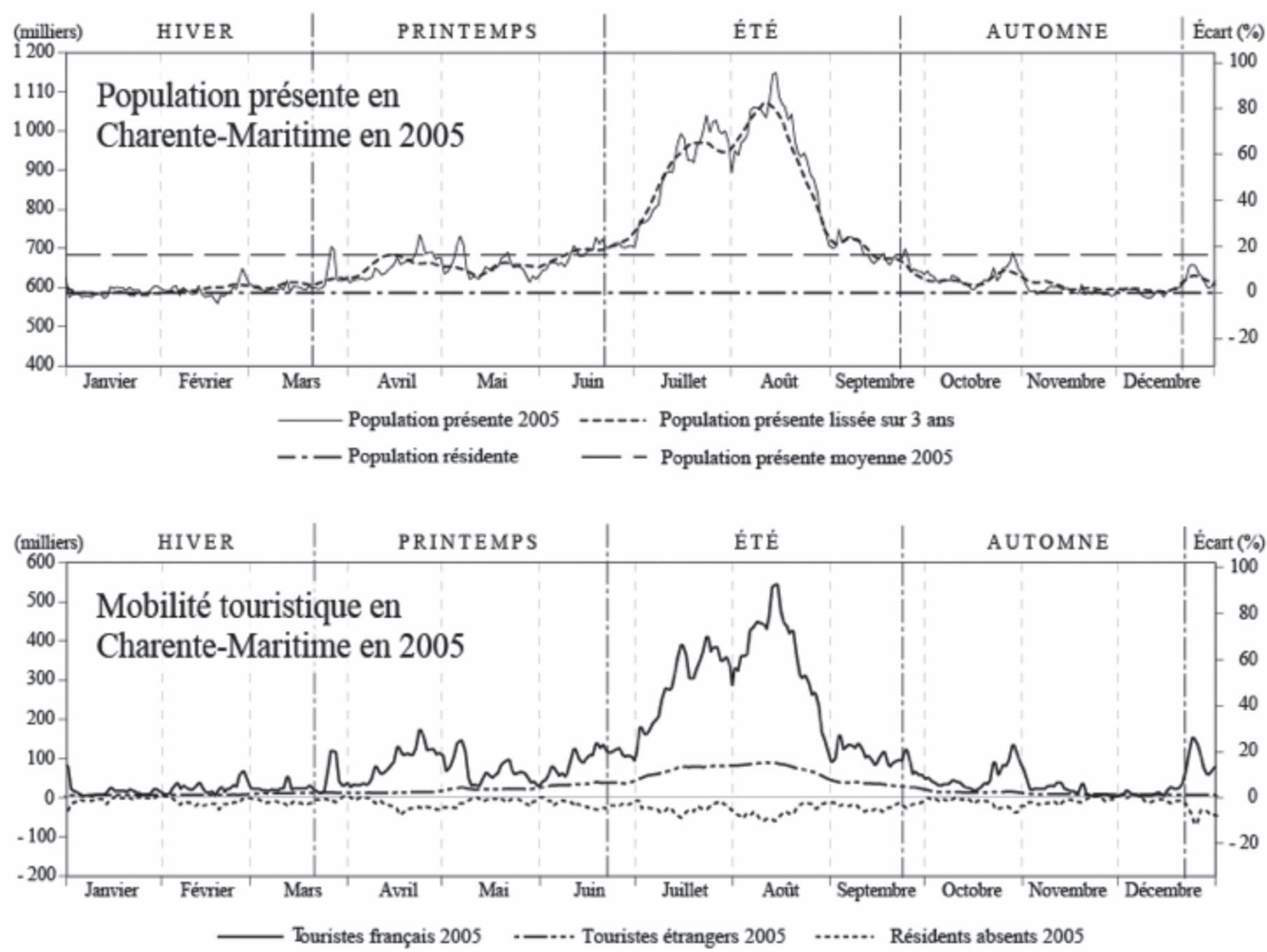

Population présente maximale, moyenne et minimale par mois en 2005

\begin{tabular}{|l|c|c|c|c|c|c|c|c|c|c|c|c|} 
& Janv & Fév & Mars & Avril & Mai & Juin & Juillet & Août & Sept & Oct & Nov & Déc \\
\hline Population présente maximale & 625600 & 648300 & 704900 & 735400 & 732600 & 727900 & 1039100 & 1148500 & 750700 & 690000 & 611800 & 659900 \\
\hline Population présente moyenne & 590800 & 593600 & 610500 & 657700 & 652300 & 689400 & 913100 & 971000 & 685700 & 628500 & 592300 & 602400 \\
\hline Population présente minimale & 573700 & 560000 & 590000 & 611400 & 612900 & 643400 & 733700 & 704600 & 633700 & 594600 & 581200 & 574400 \\
\hline
\end{tabular}

Source graphes et tableaux : Estimation direction du Tourisme in TERRIER C. (dir.), 2006, Mobilité touristique et population présente les bases de l'économie présentielle des départements, Paris, Direction du Tourisme, 128 p.

Figure 1 : Mobilité touristique et population présente en Charente-Maritime en 2005

Tourist mobility and present-in-area population in Charente-Maritime in 2005 
férentes études de fréquentation commandées par les élus des îles d'Oléron (Duvat, 2010) ou de Ré (Guérineau, 2010) en partenariat avec l'université de La Rochelle.

Enfin, la thèse de M. Talandier (2007) dirigée par L. Davezies propose ainsi un calcul de ces populations à l'échelle communale grâce une extrapolation statistique : les départs de touristes de chaque département sont ventilés entre les communes de résidence selon les caractéristiques de la population locale (notamment l'indicateur « revenu des ménages » fortement corrélés avec le taux de départ en vacances). Pour les arrivées, les touristes sont répartis au sein du département selon la capacité d'hébergement de la commune.

À partir de cette méthodologie, Laurent Davezies estime dans une étude réalisée en décembre 2008, que le taux de présence moyen sur l'année pour les 18 communes de la Communauté d'agglomération (C.A.) de La Rochelle est de $113 \%$ (Davezies 2008). C'est donc à partir de ce périmètre de référence, la Communauté d'agglomération, et non l'agglomération au sens de l'INSEE (unité urbaine), que nous estimerons la population présente à La Rochelle. En appliquant ce taux de présence à la population de la C.A. de La Rochelle au dernier recensement de 2007 (indice $100=146362$ personnes), cela représente donc un surplus de 19027 personnes permettant d'atteindre sur l'année une population présente moyenne de 165389 personnes.

Les chiffres de C. Terrier, qui tiennent compte de la saisonnalité, montrent que la population touristique du département de la Charente-Maritime augmente de $62 \%$ pour la période juillet-août par rapport à la population résidente recensée par l'INSEE (figure 1). Un « pic de fréquentation » évalué à 1148000 personnes présentes dans le département le 14 août 2005 permet presque de doubler $(+95,5 \%)$ cette population résidente.

Si l'on applique ces mêmes augmentations à la population présente moyenne de l'agglomération rochelaise calculée par Davezies, cela implique 72500 personnes supplémentaires sur la période juillet/août soit une population d'environ 220000 personnes avec un pic estival ${ }^{5}$ à 260000 personnes.

5. Ces chiffres sont obtenus en comparant le surplus de population à l'échelle départementale $(+95800)$ à celui à l'échelle de l'agglomération $(+19000)$. On peut ainsi estimer que l'agglomération de La Rochelle capte

\section{Des populations à mieux prendre en compte a l'échelle locale}

Mais aussi spectaculaire soit-il, cet apport de population est en fait sous-estimé à l'échelle locale. Il est en effet calculé à partir d'une population « présente » calculée à des échelles plus petites et qui ignore certaines catégories (figure 2).

Le surplus de population présente de C. Terrier est obtenu en faisant la différence entre des résidents absents et des touristes présents. Il ne concerne que des « séjournants ", autrement dit des populations qui passent au moins une nuit en dehors de leur domicile. Les populations en déplacement pour la journée ne sont donc pas comptabilisées. Ces populations qui fréquentent les lycées ou l'université, qui travaillent ou qui viennent visiter la ville pour la journée (excursionnistes) sont pourtant importantes pour le fonctionnement de l'économie, la justification de certains services comme pour la génération d'embouteillages.

Les pendulaires ou navetteurs par leurs déplacements quotidiens de leur domicile à leur travail génèrent une redistribution spatiale des populations durant la journée. À l'échelle de la Communauté d'agglomération, le solde entre les personnes qui quittent l'espace urbain pour la journée et ceux qui y viennent depuis l'extérieur s'élève à 29460 navetteurs d'après les chiffres INSEE sur les déplacements domicile-travail issus du Recensement de la population 2007 (exploitation complémentaire). On peut estimer qu'en juillet/août, le nombre d'actifs en vacances réduit ce chiffre de moitié.

Il faut aussi considérer les mouvements journaliers des scolaires et des étudiants. Le croisement entre les chiffres du Recensement de la population 2007 sur la population scolarisée à l'échelle communale et ceux des transports scolaires, auxquels s'ajoutent ceux des effectifs étudiants de l'université de La Rochelle nous permettent d'estimer un solde au bénéfice de la Communauté d'agglomération de La Rochelle de 3582 personnes en période scolaire. Le solde entre les entrées et les sorties de l'agglomération est faible pour les collèges (340 collégiens) dont le recrutement est local.

environ $1 / 5^{e}$ des flux supplémentaires du département et appliquer ce ratio aux données produites par C. Terrier pour le département de Charente-Maritime. 


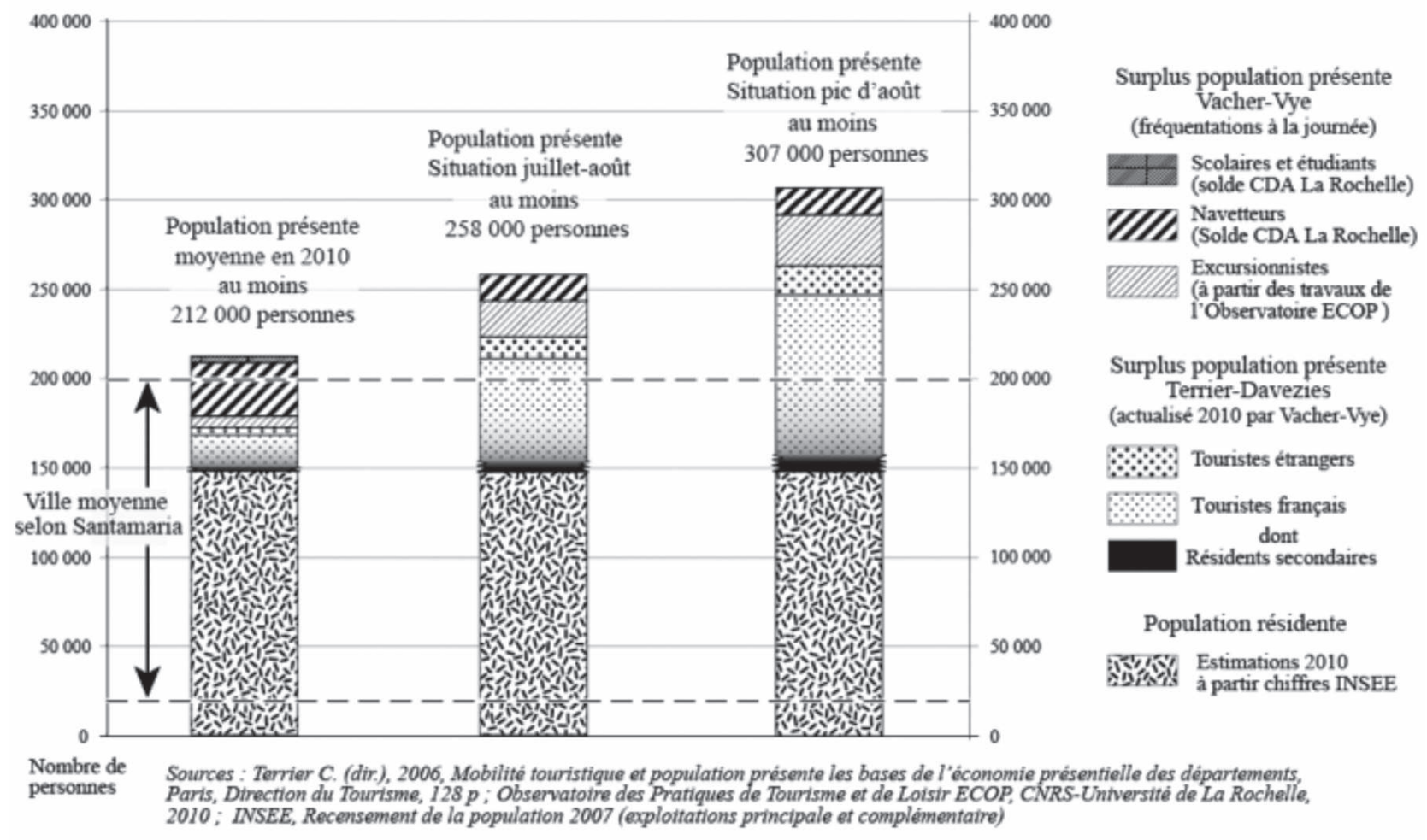

Figure 2 : Population présente estimée dans l'agglomération de La Rochelle en 2010 Estimated present-in-area population in the La Rochelle conurbation in 2010

Il est plus fort pour l'université dont 1200 personnes sont susceptibles de faire le trajet chaque jour depuis une commune du nord de la CharenteMaritime. Enfin c'est pour les lycéens du nord du département que le solde est le plus important avec 1600 lycéens extérieurs à la Communauté d'agglomération et faisant quotidiennement le trajet vers un des 10 lycées de la ville.

Enfin, il convient de comptabiliser les excursionnistes, c'est-à-dire les visiteurs à la journée pour des motifs touristiques. Les enquêtes menées sur les plages dans le cadre de l'Observatoire des pratiques de tourisme et de loisir (ECOP) nous ont permis d'identifier différents profils de populations présentes dans une ville touristique telle que La Rochelle en saison estivale (ici en 2008 et 2009). Ces profils ont été définis en fonction du statut dans le lieu : résidents principaux, résidents secondaires, touristes logés sur place en hébergement commercial ou chez des parents ou amis... (figure 3). Si une large majorité de ces populations sont prises en compte dans les méthodologies existantes de C. Terrier et de L. Davezies, les excursionnistes, qui représentent $16 \%$ des enquêtés sur les plages, ne le sont pas.

Ces excursionnistes sont absents des études de population présente, bien qu'ils soient très " présents » dans les discours où ils sont souvent accusés d'être responsables de bouchons aux ponts des îles de Ré et d'Oléron ou de «papiers gras » liés à leurs supposées pratiques abusives du piquenique, qu'ils préfèrent souvent à la restauration marchande. Or, ils forment une catégorie particulièrement bien représentée dans la ville touristique où les visites à la journée sont fréquentes. Il s'agit d'excursions pratiquées par des résidents d'une ville proche, mais aussi par des touristes en vacances à proximité : 1/4 des excursionnistes enquêtés sur l'agglomération sont en fait des touristes en vacances dans la région et qui viennent à La Rochelle pour la journée. 


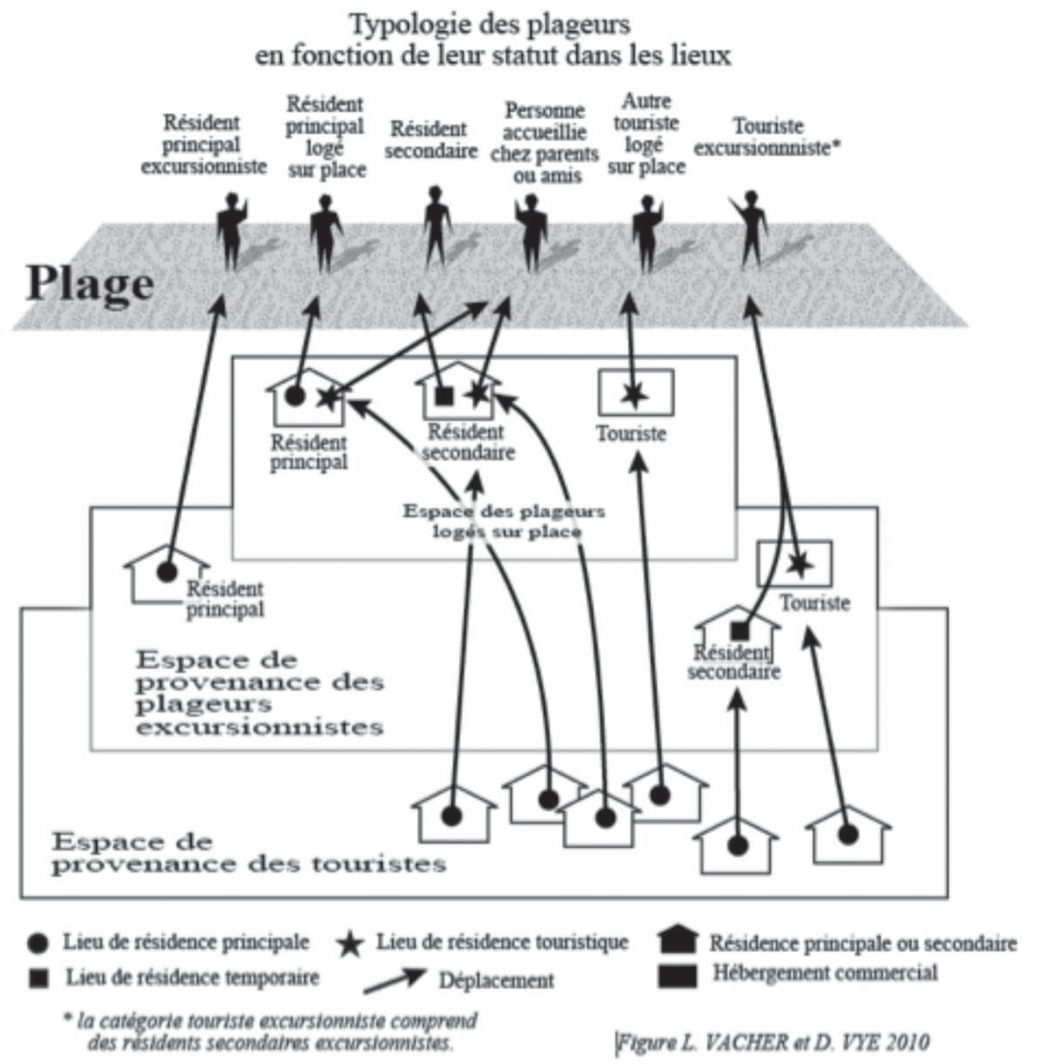

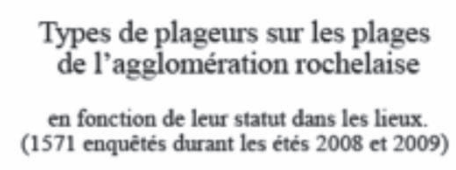

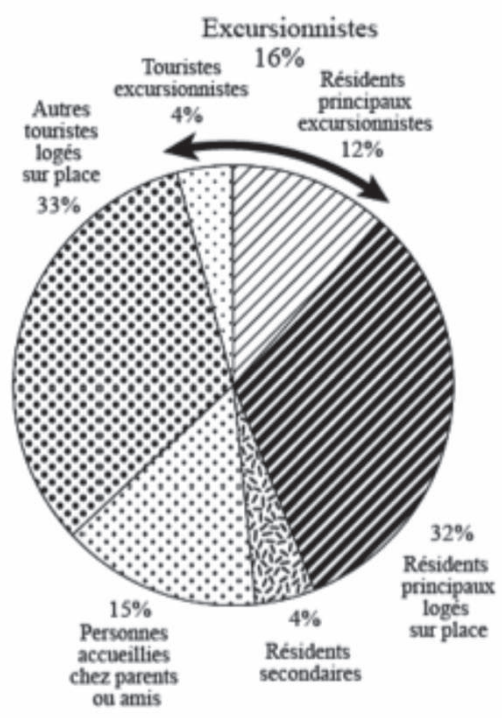
Source : Observatoire des Pratiques de Tourisme et de
Lotsir - ECOP, CNRS-Universite de La Rochelle 2010.
Figure 3 : Typologie des plageurs ${ }^{6}$ en fonction de leur statut dans les lieux et répartition par plage à La Rochelle en 2008 et 2009 Typology of beach-goers according to their status in the locations and breakdown by beach in La Rochelle in 2008 and 2009

La prise en compte de l'ensemble de ces catégories amène à revoir les estimations du surplus saisonnier pour atteindre en situation moyenne sur l'année (dépassée ou égalée durant 7 mois d'avril à octobre) au moins 212000 personnes (figure 2). Le seuil «ville moyenne » est allègrement dépassé en juillet-août avec une population présente d'au moins 258000 personnes qui atteint en période de pic 307000 personnes minimum.

On pourrait aussi ajouter les déplacements des personnes résidant à l'extérieur de l'agglomération et venant fréquenter un commerce ou un service dans la journée. Une partie d'entre eux est le fait des navetteurs qui combinent déplacement professionnel et fréquentation des commerces et services. Mais, une autre partie devrait être ajoutée à la population présente en journée dans l'agglomération : il

6. Un « plageur » est défini comme étant un usager de la plage à des fins de tourisme ou de loisir. Nous avons préféré, avec d'autres auteurs (Coëffé et Violier, 2008), ce néologisme au plus courant « plagiste » qui au sens strict désigne une personne qui loue un équipement de plage ou qui exploite une plage payante. s'agit des personnes venant profiter des infrastructures de loisir de l'agglomération (club de voile, salle de gymnastique, conservatoire, etc.).

Les estimations proposées ici sont donc minimales d'autant plus en considérant la question de la résidence secondaire dont les usages sont en général mal pris en compte. Or, cela pose problème lorsqu'on constate avec l'INSEE et le Comité Départemental du tourisme de Charente-Maritime (CDT 17) que la Communauté d'agglomération de La Rochelle offre plus de lits en résidence secondaire (27000) qu'en hébergement commercial (20000). Bien que les travaux de $\mathrm{C}$. Terrier comptabilisent les résidents secondaires dans les calculs de la population touristique, il est possible de se demander si les différents usages touristiques de l'objet « résidence secondaire » sont justement analysés (c'est pour cela qu'ils sont indiqués en accordéons sur la figure). Or ces usages sont souvent ignorés au moins du point de vue des statistiques du tourisme (Sacareau, Vacher, Vye, 2012, à paraitre). Or, les usages de la résidence secondaire concernent de nombreuses catégories d'utilisateurs avec notamment de nombreuses transactions marchandes, qu'elles soient déclarées ou non (figure 4). 

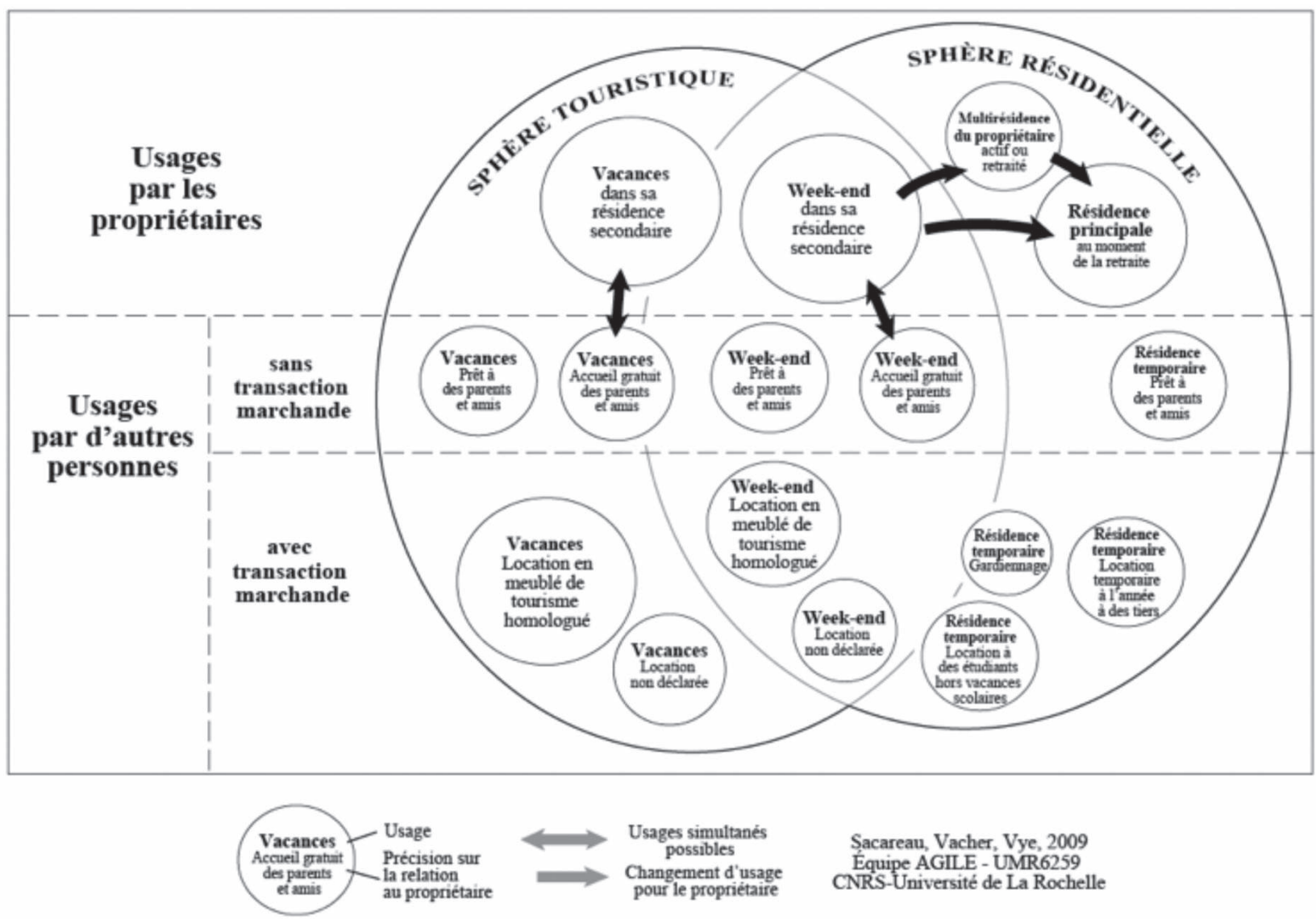

Sacareau, Vacher, Vye, 2009
Equipe AGILE - UMR6259

Equipe AGILE - UMR6259

Figure 4 : Les différents usages de la résidence secondaire

The various uses of second homes

Cependant, les seuils démographiques ne sont que des éléments partiels de définition d'une ville moyenne. Au sein de cette catégorie de ville, il convient de s'interroger également sur l'intensité d'une urbanité caractérisée par le couple densité/ diversité. L'analyse des types de populations présentes dans la ville pour des motifs liés à des pratiques de tourisme et de loisir sera l'entrée choisie pour caractériser cette urbanité.

\section{Diversité et densité de LA POPU- LATION PRÉSENTE DANS LA VILLE TOURISTIQUE : VERS UNE URBANITÉ RENFORCÉE?}

L'hypothèse retenue ici est que le caractère touristique tend à renforcer à la fois la densité, à travers notamment la notion de population présente, et la diversité d'une ville et par là même son urbanité, augmentant ainsi sa position dans la hiérarchie urbaine. Par là même, il est possible de se demander si, au sein d'une agglomération touristique littorale telle que La Rochelle, l'analyse comparative de la fréquentation des plages urbaines permet de distinguer des lieux marqués par un degré d'urbanité plus moins grand.

\section{La Rochelle : agglomération touristique}

Cependant, cette hypothèse n'est pertinente qu'à l'échelle de la ville touristique pour reprendre la terminologie mise au point par l'équipe MIT (Équipe MIT, 2003) ${ }^{7}$. En effet, dans une ville touristique, le

7. À la différence de la station touristique définie par la primauté de l'activité touristique dans un lieu, la ville touristique est un lieu non dominé par le tourisme. Celui-ci a investi et diverti (au sens de détourner, éloigner) la ville mais sans modifier fondamentalement la structure urbaine. Cette caractéristique la distingue de la ville touristifiée, investie mais aussi subvertie (au sens de bouleverser) par le tourisme et dont la structure spatiale originelle a été patrimonialisée par le tourisme, en particulier le centre qui concentre l'essentiel des flux (exemple: Venise, Bruges, Saint-Tropez, Kotor..). 
tourisme n'est qu'une fonction parmi d'autres et la taille démographique est généralement plus grande que dans d'autres types de lieux touristiques (Stock, 2001). Or, c'est bien à cette catégorie de ville qu'appartient notre terrain d'étude La Rochelle, par ailleurs ville portuaire, industrielle et plus récemment universitaire. Toutefois, le cas rochelais présente l'originalité de présenter au sein de son unité urbaine $^{8}$, plusieurs lieux touristiques répartis sur plusieurs communes (figure 5).

La ville-centre regroupe un cœur historique patrimonialisé autour du vieux-port, lieu principal de concentration des flux touristiques, le site de l'Aquarium qui attire plusieurs milliers de visiteurs par an, et un quartier, Les Minimes, organisé depuis les années 1970 autour du plus vaste port de plaisance de la façade atlantique. Les autres communes littorales de l'agglomération sont marquées par une opposition entre une côte nord à falaises au tourisme littoral négligeable et une côte sud, alternant pointes rocheuses et anses, plus attractive. Cette dernière s'organise, autour de deux communes où se côtoient campings et plages (Aytré et dans une moindre mesure Angoulins) et d'une véritable station balnéaire (Châtelaillon-Plage). Cette diversité de lieux touristiques fait de La Rochelle une véritable agglomération touristique.

\section{LA PLAGE : LIEU DE DIVERSITÉ ET DE COPRÉSENCE AU SEIN DE L'AGGLO- MÉRATION TOURISTIQUE LITTORALE}

Au sein de cette agglomération touristique, la plage est un terrain d'analyse intéressant pour évaluer l'intensité de l'urbanité. Espace public et gratuit, lieu de tourisme comme de loisir, elle est en haute saison touristique, un lieu de forte fréquentation. La fréquentation de cet espace est un marqueur de diversité dans la mesure où se côtoient dans un même lieu résidents permanents, touristes, résidents secondaires, excursionnistes, a priori de tous âges et de toutes conditions sociales. Cette diversité engendre donc des situations spatiales de

8. L'agglomération (ou Unité urbaine) au sens INSEE est ici à distinguer de la Communauté d'agglomération (EPCI - espace politique). La première comprend 8 communes (Angoulins, Aytré, Châtelaillon-Plage, Lagord, Nieul-sur-Mer, Périgny, Puilboreau et La Rochelle) quand la seconde comprend en plus les communes périurbaines de L'Houmeau, Salles-SurMer, Saint-Vivien, Marsilly, Dompierre-sur-Mer, Esnandes, Saint-Xandre, Sainte-Soulle, et Saint-Rogatien) - figure 5. coprésence, c'est-à-dire une présence simultanée dans un lieu de populations différenciées. Enfin, la plage, dans une agglomération touristique, reproduit également les rythmes de la ville dont elle n'est finalement qu'un prolongement.

Dans le cadre de l'Observatoire des pratiques de tourisme et de loisir', les plages de l'agglomération rochelaise étudiées depuis 2008 durant la saison estivale présentent différentes configurations (figure 5) : deux d'entre elles, sont situées à proximité du cœur touristique de l'agglomération (plage de la Concurrence proche du Vieux port et du centre historique, plage des Minimes à proximité du port de plaisance). Une troisième en tant que plage de station est un lieu d'attraction à elle seule (plage de Châtelaillon). Une quatrième, la plage d'Aytré, en position intermédiaire entre ces deux pôles, peut apparaître comme un lieu touristique secondaire lié à la présence de campings. Enfin, la plage de Chefde-Baie, non loin de la zone industrialo-portuaire de La Pallice, est la seule plage étudiée à ne pas être située à proximité d'un lieu touristique.

Ces différentes plages accueillent une population de « plageurs » qui peuvent se différencier selon une multitude de critères (sexe, âge, CSP etc.). Dans le cadre de cet article, nous avons privilégié la question du statut dans le lieu (voir figure 3). Ce choix permet en effet de mettre en avant des catégories non identifiées parmi les populations présentes comme les excursionnistes (voir 1 re partie) et d'autre part de traiter la question de l'attractivité de la ville touristique (voir 3e partie). Cette catégorisation tranche avec la plupart des recherches sur le tourisme qui résument généralement les profils des personnes qui fréquentent un lieu touristique à une simple opposition entre «touristes » et " population locale ». De plus, les statistiques de fréquentation touristique ne traitent souvent que des touristes hébergés dans le secteur marchand.

Or, selon cette approche selon le statut dans le lieu, nous avons pu identifier 6 catégories en fonc-

\footnotetext{
9. Dans le cadre de cet observatoire, plus de 5600 questionnaires ont été recueillis entre 2008 et 2011 sur une sélection des principales plages du nord de la Charente-Maritime (voir figure 6). Ces questionnaires ont été administrés directement sur le lieu de pratique, la plage, et ce en pleine saison estivale. Pour la campagne 2009, citée dans cet article, les questionnaires ont été réalisés entre le 15 juillet et le 10 août avec au final 1198 individus interrogés sur l'ensemble des 8 plages enquêtées.
} 


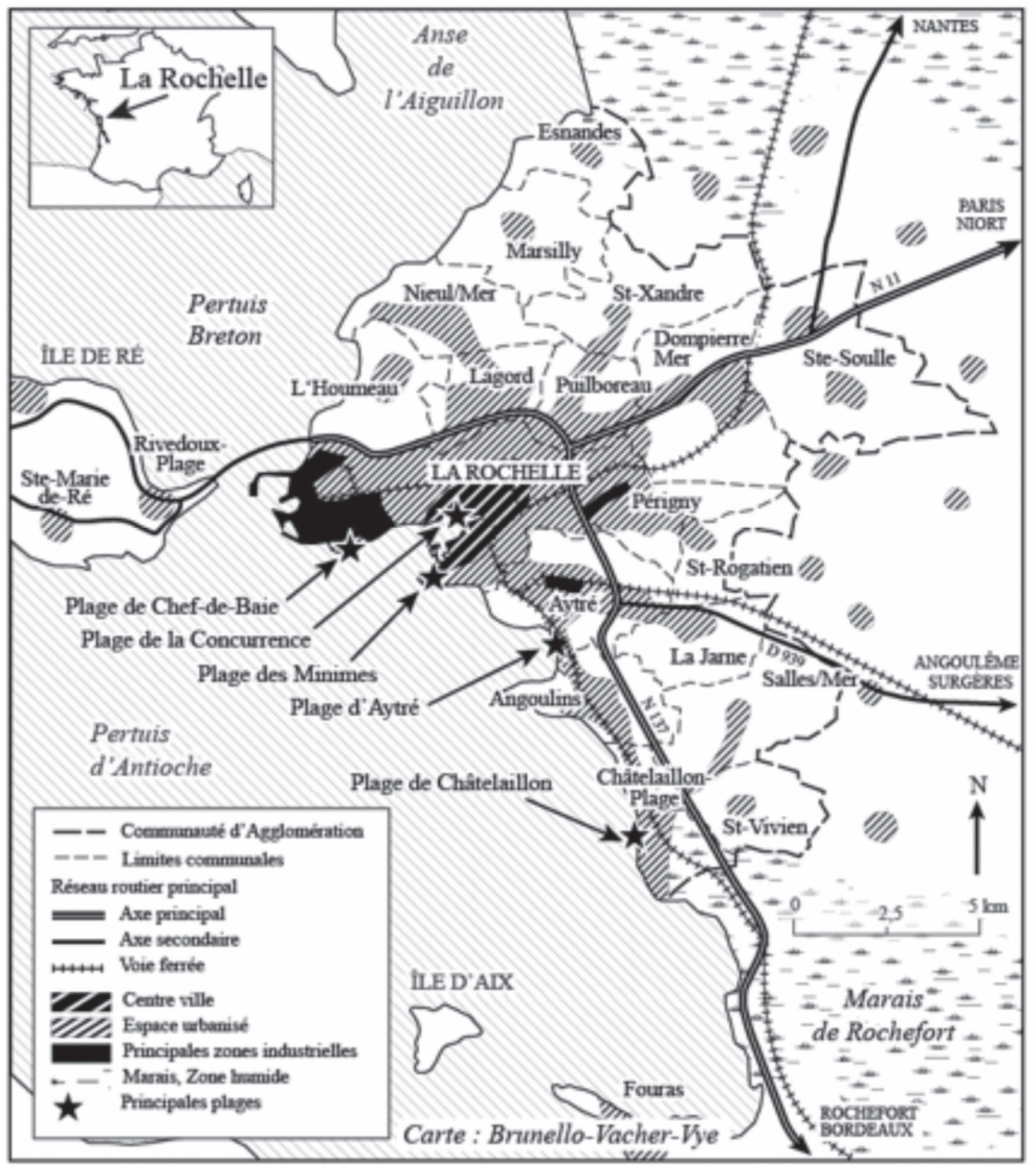

Figure 5 : Localisation des plages étudiées au sein de l'agglomération rochelaise

Location of beaches surveyed in the La Rochelle conurbation tion de trois critères de distinction différents ${ }^{10}$ (figure 6) :

- le fait d'être touriste ou résident principal;

- le fait d'être logé sur place - au sein de la Communauté d'agglomération de La Rochelle - ou excursionniste;

- le fait d'être, en tant que touriste, hébergé chez des parents ou amis ou logé en hébergement marchand.

10. Une catégorie supplémentaire, les travailleurs saisonniers, ni touristes, ni habitant permanent du lieu a été introduite en 2009. Relativement présente sur l'Ile de Ré voisine, elle ne représente toutefois qu'à peine 1 \% des enquêtés à La Rochelle. Nous avons donc choisi de ne pas en tenir compte ici.
Or, ces six catégories ne fréquentent pas avec la même intensité les différentes plages de l'agglomération (figure 6).

Logiquement, en saison estivale, les touristes sont globalement plus nombreux que les résidents principaux. Toutefois, les plages de l'agglomération rochelaise, à la différence de la majorité des plages rétaises voisines également étudiées dans le cadre de l'Observatoire, ne comptent aucune plage spécifiquement touristique, c'est-à-dire comptant plus de $50 \%$ de touristes en hébergement marchand ${ }^{11}$.

11. Les plages spécifiquement touristiques sont caractérisées par une large majorité de touristes logeant sur place en hébergement marchand ( 2 plageurs sur 3 à Trousse-Chemise, 3 sur 4 au Bois-Plage) et dans une 

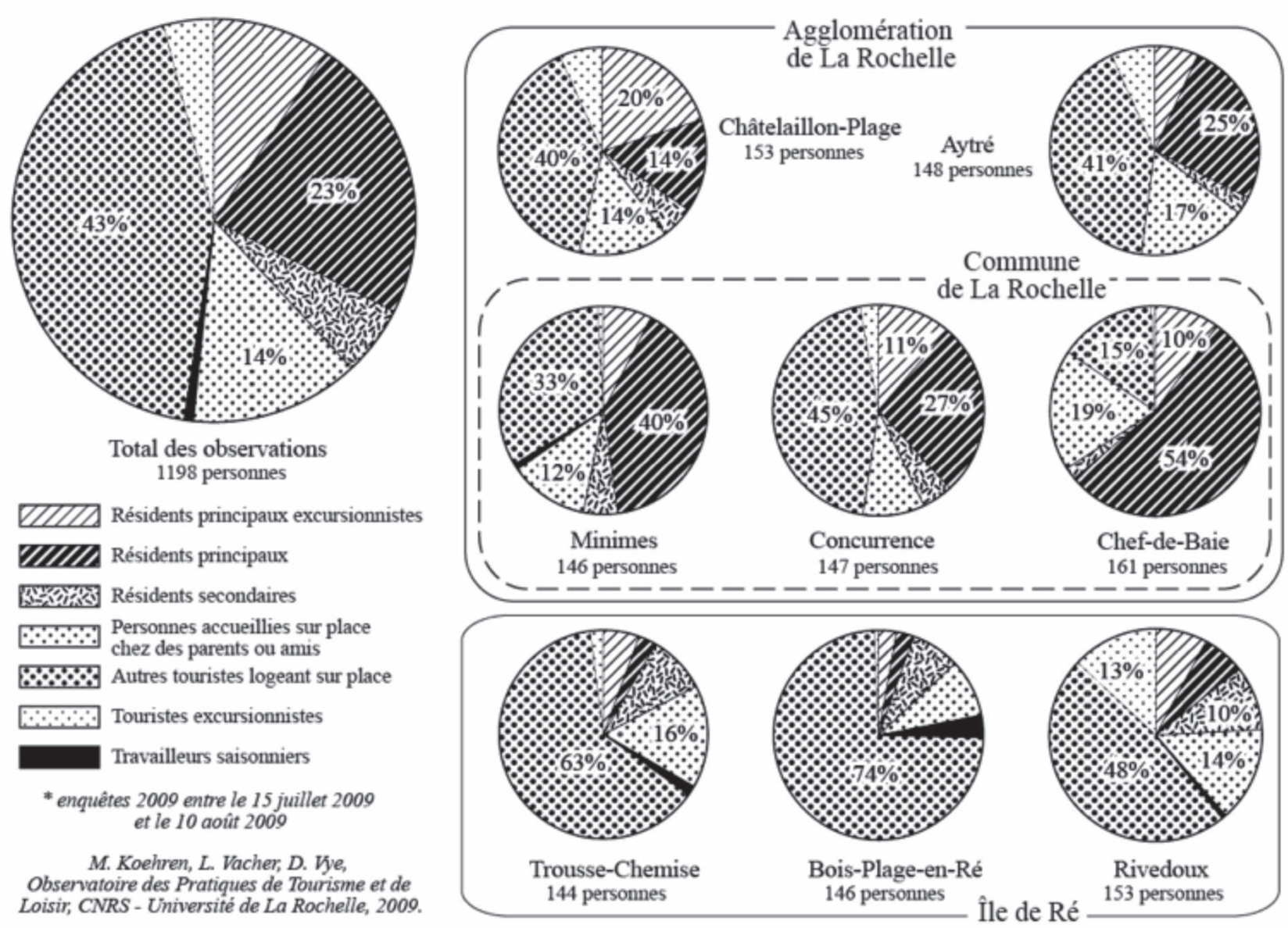

Figure 6 : Statut des personnes présentes sur quelques plages de l'agglomération de La Rochelle et de l'île de Ré durant l'été 2009 Status of people present on certain beaches in the La Rochelle conurbation and on Île de Ré during summer 2009

En effet, sur les plages étudiées se côtoient des populations touristiques de natures différentes: si les touristes en hébergement marchand restent la catégorie la plus représentée à l'échelle de l'agglomération, notamment sur les plages de La Concurrence, Châtelaillon ou Aytré, on note la présence non négligeable de personnes accueillies chez des parents ou amis, de résidents secondaires ou de touristes excursionnistes. Cela explique pourquoi aucune de nos plages ne dépassent les $50 \%$ de touristes en hébergement marchand.

À côté de cette population touristique, les résidents principaux habitant la Communauté d'agglomération sont également très présents : ils représentent un plageur sur trois à l'échelle de l'agglomé-

moindre mesure par des touristes logés dans leur propre résidence secondaire ou dans celle d'amis ou de parents. La part des résidents principaux tout comme celle des excursionnistes y est en revanche négligeable. ration soit autant que les touristes en hébergement marchand et sont la catégorie la plus nombreuse aux Minimes et sont mêmes majoritaires à Chefde-Baie.

Enfin, les excursionnistes complètent cet éventail de plageurs. Ils sont essentiellement des résidents permanents, habitant des centres urbains proches du nord de la région Poitou-Charentes (Niort, Poitiers...) et venant passer une journée à la plage. Ils sont particulièrement nombreux à Châtelaillon $(20 \%)$.

Or, la répartition entre ces différentes catégories est inégale d'une plage à une autre. Il est alors possible de distinguer des critères de diversité (voir tableau 1) selon les plages. Celles présentant la plus forte diversité sont celles qui ont une fréquentation « équilibrée » : elles accueillent une part importante mais non dominante de touristes en hébergement marchand 


\begin{tabular}{|c|c|c|c|c|c|c|c|c|}
\hline \multirow[b]{2}{*}{ Type } & \multirow[b]{2}{*}{ Plage } & \multicolumn{4}{|c|}{ Critères de diversité } & \multicolumn{2}{|c|}{ Critères de densité } & \multirow[b]{2}{*}{ Urbanité } \\
\hline & & $\begin{array}{c}\text { Entre } 30 \text { et } \\
50 \% \\
\text { de touristes } \\
\text { en héberge- } \\
\text { ment mar- } \\
\text { chand }\end{array}$ & $\begin{array}{c}\text { Entre } 25 \text { et } \\
45 \% \\
\text { de résidents } \\
\text { principaux }\end{array}$ & $\begin{array}{c}\text { Entre } 10 \text { et } \\
20 \% \\
\text { d'excursion- } \\
\text { nistes }\end{array}$ & Diversité & $\begin{array}{c}\text { Densité } \\
\text { (nombre de } \\
\text { m2 par } \\
\text { plageur) }\end{array}$ & Densité & \\
\hline $\begin{array}{l}\text { Plage de } \\
\text { centralité } \\
\text { urbaine }\end{array}$ & $\begin{array}{c}\text { Minimes } \\
\text { Concurrence }\end{array}$ & Oui & Oui & Oui & +++ & $\begin{array}{c}\text { Forte } \\
\left(5 \text { à } 10 \mathrm{~m}^{2}\right)\end{array}$ & ++ & Trés forte \\
\hline $\begin{array}{c}\text { Plage } \\
\text { périurbaine }\end{array}$ & Aytré & Oui & Oui & Oui & +++ & $\begin{array}{l}\text { Moyenne } \\
\left(10 \text { à } 25 \mathrm{~m}^{2}\right)\end{array}$ & + & Forte \\
\hline $\begin{array}{l}\text { Plage de } \\
\text { Station }\end{array}$ & Châtelaillon & Oui & $\begin{array}{c}\text { Non } \\
\text { (- de } 25 \%)\end{array}$ & $\begin{array}{c}\text { Non } \\
(+\operatorname{de} 25 \%)\end{array}$ & + & $\begin{array}{l}\text { Moyenne } \\
\left(10 \text { à } 25 \mathrm{~m}^{2}\right)\end{array}$ & + & Moyenne \\
\hline $\begin{array}{l}\text { Plage de } \\
\text { citadins }\end{array}$ & Chef-de-Baie & $\begin{array}{c}\text { Non } \\
\text { (- de } 30 \%)\end{array}$ & $\begin{array}{c}\text { Non } \\
(+ \text { de } 50 \%)\end{array}$ & $\begin{array}{c}\text { Non } \\
\text { (- de } 10 \%)\end{array}$ & - & $\begin{array}{l}\text { Trés forte } \\
\left(- \text { de } 5 \mathrm{~m}^{2}\right)\end{array}$ & +++ & Moyenne \\
\hline
\end{tabular}

Tableau 1 : Typologie des plages de l'agglomération rochelaise selon leur degré d'urbanité (Source : Observatoire des pratiques de tourisme et de loisir, enquêtes été 2009)

Typology of beaches in the La Rochelle conurbation according to their degree of urbanity

(entre 30 et $50 \%$ ), combinée à une part notable de résidents principaux (entre 25 et $45 \%$ ) et une part non négligeable d'excursionnistes (entre 10 et $20 \%$ ). Cela concerne trois plages de l'agglomération rochelaise : les Minimes, la Concurrence et Aytré.

Toutefois, les seuls critères de la diversité s'avèrent nécessaires mais non suffisants pour évaluer le degré d'urbanité. Il faut alors les croiser avec celui de la densité.

\section{DES TYPES D'URBANITÉ VARIABLES SELON LES PLAGES}

Nous avons pu identifier 4 types d'urbanité et ce, en fonction de critères qui combinent diversité et densité (tableau 1). Les données relatives à la densité sont exprimées en nombre de $\mathrm{m}^{2}$ par plageur (surface disponible) et sont issues de la campagne d'observation aérienne 2009 réalisée en parallèle des enquêtes qualitatives.

Les plages de centralité urbaine (Minimes et Concurrence) sont celles qui présente la plus forte urbanité. Elles sont en effet marquées par une forte densité en saison estivale liée à la coprésence de catégories variées. Elles sont le prolongement du pôle touristique, économique et résidentiel de l'agglomération. Ainsi, la Concurrence est fréquentée par des touristes, des résidents principaux et des excursionnistes en raison de sa proximité avec le centre-ville historique qui combine fonction touristique, de loisir, d'hébergement mais également de travail. En effet, la plage de la Concurrence est celle qui présente le plus fort taux de résidents permanents qui travaillent $41 \%$ en 2009). Ces derniers sont ainsi nombreux à fréquenter la plage à l'heure de la pause de midi ou après une journée de travail. Aux Minimes, la plage possède la spécificité d'accueillir davantage de résidents principaux en vacances, de résidents secondaires ou de personnes accueillies chez des parents ou amis.

Aytré est une plage à la fois périphérique et urbaine, d'où son qualificatif de plage périurbaine : périphérique en raison de sa situation géographique par rapport à la ville-centre et de sa plus faible densité de plageurs; urbaine par la diversité de sa fréquentation et par sa part notable de résidents principaux (1/4). Parmi ces derniers, comme à La Concurrence, une part importante (33\%) n'est pas en vacances.

L'urbanité de la plage de Chef-de-Baie se caractérise avant tout par une densité très forte (moins 
de $5 \mathrm{~m}^{2}$ disponible par plageur). En revanche, sa fréquentation est peu diversifiée, constituée dans une large mesure (54\%) par les habitants de l'agglomération. Nous la qualifierons donc de plage de citadins. La fréquentation touristique n'est toutefois pas absente mais les touristes sont davantage hébergés chez des parents ou amis (19\%) que dans des formes classiques d'hébergement marchand (15\%). On peut émettre l'hypothèse que ce sont ces mêmes parents ou amis qui leur ont fait découvrir une plage peu connue, car plus excentrée mais offrant la possibilité, unique dans l'agglomération, d'une baignade quelle que soit la marée. C'est donc une plage dont la dimension touristique est ici indirecte, dans la mesure où elle semble corrélée aux pratiques de loisirs des Rochelais qui fréquentent cette plage dans leur espace-temps du quotidien.

Enfin, la plage de Châtelaillon est une plage de station mais sans être spécifiquement touristique tout en étant marquée par une densité moyenne. Son originalité réside dans le fait que près d'un plageur sur trois (31\%) est un excursionniste, qu'il soit touriste ou résident principal. De plus, durant l'été en tout cas, c'est une plage peu fréquentée par les habitants de l'agglomération.

Parce qu'elles figurent parmi les principaux lieux fréquentés par les populations présentes pour des motifs de tourisme ou de loisir, les plages sont des supports intéressants pour mieux cerner l'urbanité d'une ville touristique littorale. De plus, ces espaces publics sont eux-mêmes marqués par des degrés d'urbanité plus ou moins importants. Ainsi, la déclinaison de ces différentes formes d'urbanité montre la complexité des modalités de fréquentation d'une agglomération touristique telle que La Rochelle. La diversité des statuts dans le lieu, associée à des formes de densités temporairement élevées en particulier sur les plages les plus centrales de l'agglomération, montre donc bien qu’à des degrés divers, la plage permet de caractériser le haut niveau d'urbanité d'une ville moyenne touristique.

\section{LA PROXIMITÉ ENTRE LA VILLE MOYENNE TOURISTIQUE ET LA MÉTROPOLE}

Au-delà de cette urbanité renforcée, n’y aurait-il pas des formes de proximité entre la ville moyenne touristique et la métropole dans le sens où d'une part une ville touristique posséderait une attractivité d'ampleur comparable à celle d'une métropole et d'autre part, parce que son attractivité touristique et résidentielle comporterait une forte composante métropolitaine?

\section{Une attractivité digne d'une métropole?}

À l'image d'autres hauts-lieux du tourisme, l'attractivité touristique de l'agglomération rochelaise mesurée à partir des enquêtes menées sur les plages en 2010 montre que l'aire d'attraction touristique de la ville est nationale avec une forte représentation des centres urbains les plus importants : il n'y a guère de vide ou de surreprésentation forte (figure 7). Néanmoins, il se dégage une sorte de faisceau principal de convergence des flux s'ouvrant vers le nord et l'est et soulignant la relative discrétion des touristes en provenance des régions du sud, mais aussi de Bretagne et de Normandie. De plus, il n'est pas possible de dessiner un gradient de la proximité par l'examen de l'origine des touristes français : les régions Rhône-Alpes ou NordPas-de-Calais attirent plus que l'Aquitaine ou la Bretagne. Notons également que même si la région Île-de-France est la principale région émettrice de touristes vers l'agglomération, son poids reste raisonnable au regard de son importance économique et démographique. L'inscription de l'aire d'attraction se fait donc nettement à l'échelle nationale et non à l'échelle régionale. La fonction touristique inscrit ainsi La Rochelle dans un schéma d'attractivité à l'échelle nationale voir internationale avec une représentation notable des touristes étrangers principalement en provenance de Belgique, Pays-Bas, Royaume-Uni et Allemagne.

L'attractivité peut aussi se penser à une échelle plus locale avec l'espace de provenance des excursionnistes (figure 8). Il s'agit là des lieux de vie des résidents principaux se déplaçant pour la journée, mais aussi des lieux de séjour des touristes hébergés à proximité et qui décident de venir passer la journée à La Rochelle dans le cadre de leur séjour touristique. Un espace local très net se dessine englobant la plus grande partie des excursionnistes dans un rayon de $50 \mathrm{~km}$ autour de la ville. Au-delà, la représentation est beaucoup plus dispersée et devient anecdotique en dehors de la région PoitouCharentes. Cette situation peut paraître étrange 


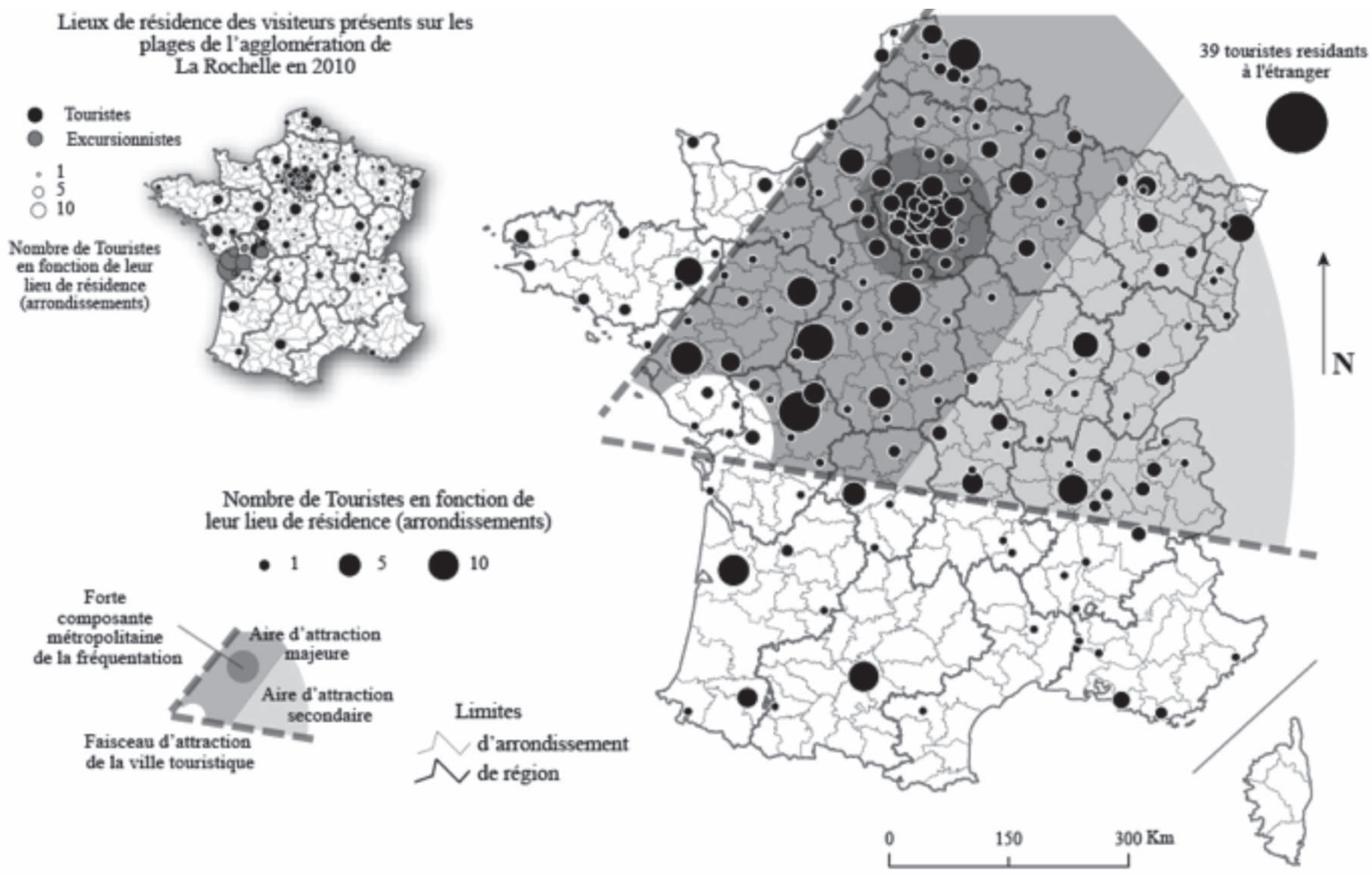

Figure 7 : Lieux de résidence des touristes présents sur les plages de l'agglomération de La Rochelle durant l'été 2010 Places of residence of tourists present on the beaches of the La Rochelle conurbation during summer 2010

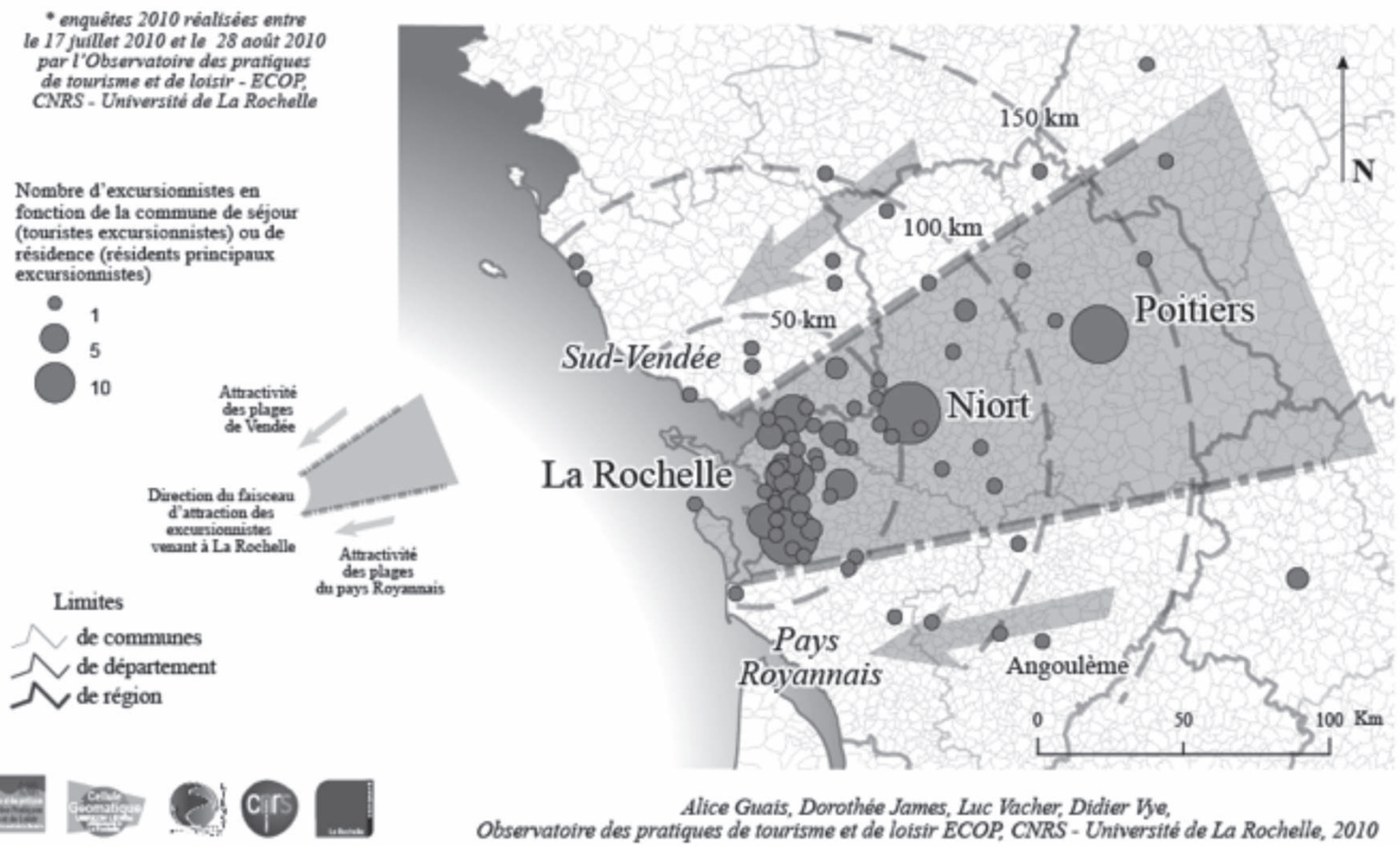

Figure 8 : Lieux d'origine des excursionnistes présents sur les plages de l'agglomération de La Rochelle durant l'été 2010 Places of origin of day-trippers present on the beaches of the La Rochelle conurbation during summer 2010 
quand on sait que la ville se trouve à quelques kilomètres du département de la Vendée qui fait partie de la région voisine des Pays de la Loire. Elle renvoie à la logique du faisceau qui apparaît ici comme encore plus étroit. La présence de lieux touristiques avec de nombreuses plages réputées en Vendée au nord et dans le Pays royannais au sud explique cette compression de l'aire d'attraction. L'aire d'attraction des excursionnistes serait donc beaucoup plus conforme à ce que l'on attend d'une ville moyenne dont l'attractivité est rapidement concurrencée par celles d'autres pôles de taille ou de fonctions équivalentes. Cette observation doit cependant être relativisée par le fait qu'au-delà de cette attraction locale, la ville génère aussi des flux régionaux non négligeables en provenance de Poitiers et de Niort, deux autres villes importantes de la région. La logique du faisceau est cependant là encore respectée : les flux en provenance d'une ville comme Angoulême, presque aussi proche de La Rochelle que Poitiers, mais se situant au sud du faisceau, sont négligeables. Cette logique, qui peut apparaître comme en lien avec le réseau autoroutier et la liaison TGV, éclaire aussi la bonne tenue de la région Centre comme aire de provenance des touristes à La Rochelle.

La fonction touristique entraine donc clairement à travers les aires d'attraction des touristes et des excursionnistes des perturbations dans la lecture de l'attractivité d'une ville moyenne. L'attractivité de la ville touristique de La Rochelle qui fonctionne à toutes les échelles renvoie donc plus à une attractivité de métropole qu'à celle d'une ville moyenne, même si la logique du faisceau relativise ce constat.

\section{La composante parisienne et francilienne de l'attractivité rochelaise}

Par son poids démographique et économique inégalé, l'île-de-France est logiquement un pôle émetteur de touristes incontournable quel que soit le lieu touristique en France. Ainsi, en CharenteMaritime, selon une enquête réalisée par le Comité Départemental du Tourisme en 2004, 1 touriste sur $5(21 \%)$ était un Francilien, c'est-à-dire une personne habitant en Île-de-France (Comité Départemental du Tourisme 17, 2005).

Or, cette même enquête montre que cette part est d'autant plus forte que le territoire touristique est littoral et urbain : si la part des franciliens est plutôt faible à l'intérieur des terres (15\%), celle-ci s'élève à $22 \%$ sur le littoral pour atteindre la valeur maximale de $25 \%$ à La Rochelle et ses environs ${ }^{12}$.

Cette spécificité se retrouve à l'échelle des plages de l'agglomération rochelaise, la part des franciliens semblant corrélée aux types d'urbanité définis par ailleurs (2-3) (figure 9). Les plages de centralité urbaine (Minimes, Concurrence) sont celles qui accueillent le plus de franciliens à la différence de la plage de citadins (Chef-de-Baie) où ces derniers représentent moins d'un plageur sur 10. Les plages de centralité urbaine sont aussi celles qui accueillent le plus de Parisiens ${ }^{13}$, en particulier La Concurrence, la plage la plus proche du centre historique (Vieux Port) où près d'un Francilien sur $2(42 \%)$ est un Parisien. Ainsi, le centre-ville de La Rochelle comme Paris intra-muros sont deux lieux de centralité et de forte urbanité connectés par le tourisme, l'un d'une métropole mondiale, l'autre d'une ville touristique littorale.

De plus, cette population touristique est particulièrement fidèle : selon les enquêtes 2008 et 2009, $50 \%$ des franciliens fréquentant les plages de l'agglomération déclarent y venir régulièrement contre $42 \%$ pour les touristes originaires des autres régions françaises. Parmi les plageurs les plus fidèles à La Rochelle, figurent bien évidemment les résidents secondaires ${ }^{14}$, catégorie au sein de laquelle les franciliens sont surreprésentés : 44,5\% des résidents secondaires enquêtés entre 2008 et 2010 ont une résidence principale en Île-de-France.

Ainsi, cette régularité de la fréquentation renforce ainsi la proximité entre Paris et La Rochelle, et plus largement entre la métropole et la ville touristique. Cette proximité se vérifie également pour les nouveaux résidents permanents corroborant ainsi le lien entre attractivité touristique et attractivité résidentielle (Sacareau, Vacher, Vye, 2010). Si La Rochelle devient à partir des années 1990, une des villes les plus attractives de France, c'est en grande partie en

12. Cet espace comprend également l'île de Ré voisine. Les chiffres de l'observatoire de l'Université de La Rochelle donnent des valeurs proches : en 2009, si $16 \%$ des 1571 plageurs interrogés possédaient leur commune de résidence en Île-de-France (voir figure 9), cette proportion montait à $23 \%$ en considérant uniquement les touristes et excursionnistes. En 2010, elle s'élevait même à $31 \%$ pour les touristes en hébergement marchand.

13. Le terme de Parisien est ici entendu au sens d'habitant de la commune de Paris.

14. En 2010, 78,5 \% d'entre eux déclarent venir régulièrement à La Rochelle soit le taux le plus élevé de l'ensemble des catégories de plageurs. 


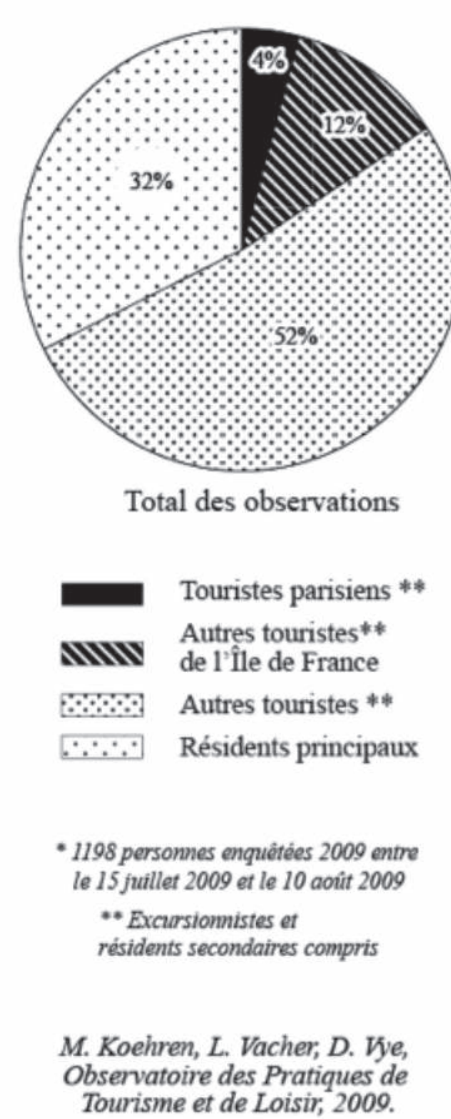

Tourisme et de Loisir, 2009.

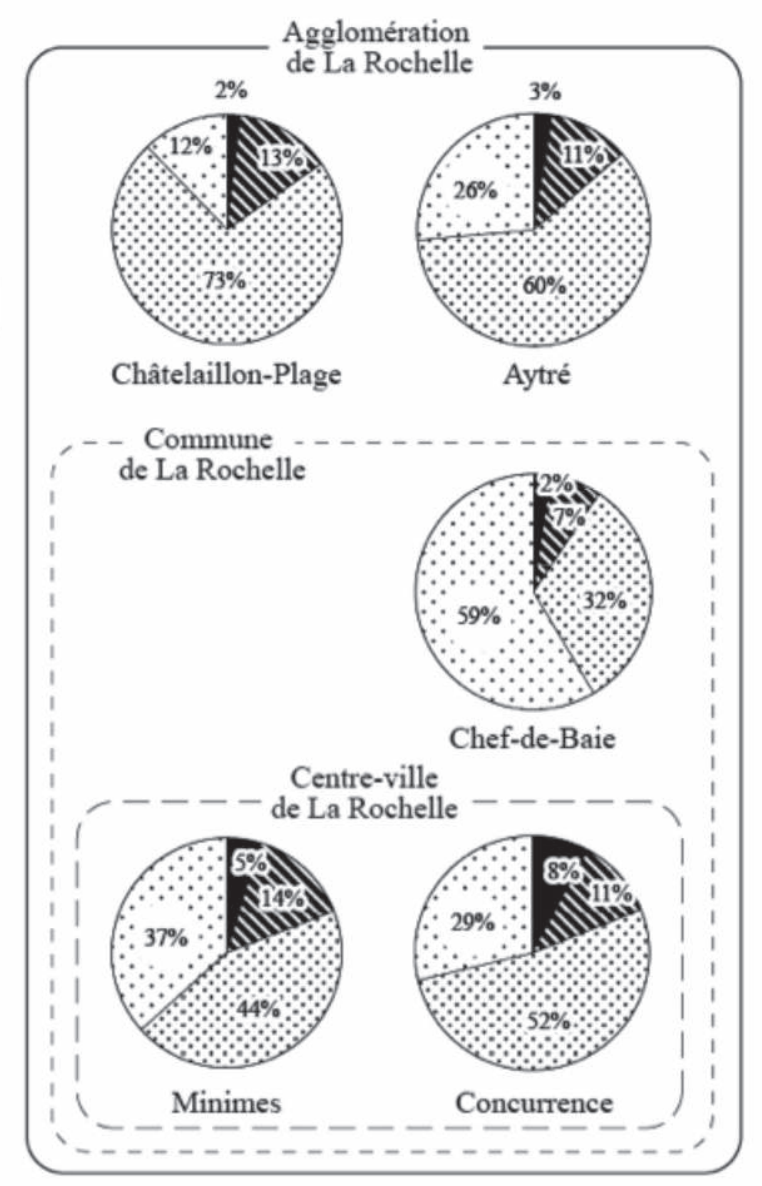

Figure 9: La part des Parisiens et des Franciliens parmi les plageurs de l'agglomération de La Rochelle durant l'été 2009. The proportion of residents of Paris and the Paris region among beach-goers in the La Rochelle conurbation during summer 2009. raison d'échanges migratoires particulièrement favorables avec l'île-de-France (Vye, 2005) ${ }^{15}$. En cela, la ville touristique littorale se démarque clairement de la capitale régionale Poitiers, " grande » ville moyenne, dont l'attractivité, pourtant globalement comparable avec celle de La Rochelle, est d'une part, essentiellement régionale et d'autre part, marquée par des échanges migratoires déficitaires avec la région parisienne.

Ainsi, La Rochelle apparaît comme une ville touristique habitée plus ou moins temporairement par des populations d'origine métropolitaine, ce qui va avoir pour effet d'accentuer l'urbanité de la ville moyenne touristique.

15. Cette logique issue de l'analyse des chiffres de la période intercensitaire 1990-1999 semble se confirmer durant la décennie suivante. Selon les chiffres INSEE du recensement version 2007, l'agglomération de La Rochelle a attiré 3490 nouveaux résidents qui habitaient lîle-deFrance, 5 ans auparavant sur un total de 14985 personnes originaires d'une autre région française, soit près d'un migrant sur quatre $(23,28 \%)$.

\section{Conclusion}

À travers une analyse des populations présentes, notamment dans des espaces publics comme les plages urbaines, nous avons pu montrer que les caractéristiques de la fréquentation, l'intensité du couple densité/diversité, l'ampleur de l'attractivité et la proximité avec la métropole étaient de nature à faire d'une ville touristique telle que La Rochelle davantage qu'une ville moyenne.

Cette fonction touristique participe de plus à produire une « image » rayonnante de la ville qui contribue à majorer sa lisibilité. Dans le cas de La Rochelle, la réputation d'une ville historiquement engagée « en faveur de l'environnement» (vélos et véhicules électriques en libre service, tri sélectif dès les années 1970), active culturellement (Festival International du film, Francofolies, Scène Nationale) et marquante dans l'événementiel (Grand Pavois, Velux5oceans, Marathon, Festival de 
la fiction TV, Université d'été du Parti Socialiste) sont autant d'éléments qui participent à la fabrication d'une image de ville dynamique et soucieuse de sa qualité de vie. Cette image qui se construit en grande partie par les flux touristiques qu'elle génère, participe à la définition d'une attractivité plus globale de la ville. Le tourisme permet donc autant la génération de l'image positive de la ville que la diffusion de cette image à l'extérieur.

L'image touristique permet à la ville moyenne de revendiquer, en toute légitimité une place qui est celle qu'elle occupe dans l'imaginaire national et explique pourquoi certains élus locaux de villes moyennes intègrent sans complexe la Fédération des Maires de Grandes Villes de France. C'est bien sûr le cas de l'agglomération de La Rochelle, mais également celui d'autres villes moyennes touristiques comme Bourges, Lorient ou la conurbation basque (Bayonne-Anglet-Biarritz).

Toutefois, la référence à la ville moyenne n'est pas abandonnée pour autant. Plutôt qu'au «médiocre », le terme «moyen » est alors associé au discours sur la ville à taille « humaine ", « harmonieuse » et « équilibrée ». À La Rochelle, cette idée est particulièrement présente dans le discours des nouveaux résidents (Vye, 2005) comme dans la rhétorique des élus ${ }^{16}$. Elle valorise alors une ville du « juste milieu », c'est-à-dire plus animée que le bourg ou la petite ville mais plus calme et conviviale que la métropole.

\section{Bibliographie}

Coëffé V., Violier P., 2008. Les lieux du tourisme : de quel(s) paradis parle-t-on?, Articulo-revue de sciences humaines, $\mathrm{n}^{\circ} 4$. Mis en ligne le 4 octobre 2008, consulté le 8 novembre 2010.

Comité departemental du tourisme 17, 2005. La clientèle francilienne, fiche 07, Observatoire du tourisme, 4 p. disponible en ligne. URL : http://www.observatoire.en-charentemaritime.com/sites/observatoire.en-charente-maritime.com/ files/FICHE-07-iledefrance.pdf. Consulté le 25 octobre 2010.

Davezies L., 2008, Les moteurs du développement de la zone de La Rochelle et de ses territoires, Poitiers, DREAL PoitouCharentes, $15 \mathrm{p}$.

16. Le Projet d'Aménagement et de Développement Durable du Schéma de Cohérence Territoriale de la Communauté d'agglomération adopté en juin 2010 affirme comme finalité le « développement raisonné et équilibré du territoire rochelais».
Davezies L., 2009. L'économie locale « résidentielle », Géographie Économie Société, volume 11, nº 1, janvier-mars 2009.

Duvat V., 2010. Étude de fréquentation et de perception de la qualité des plages, île d'Oléron : résultats de la campagne 2009. Rapport de résultats n³, Programme de recherche QUALIPLAGES, 107 p.

Équipe MIT, 2003. Le tourisme : acteurs, lieux et enjeux, Paris, Belin, 320 p.

Guérineau B., 2010. Analyse de la fréquentation et des pratiques sur l'île de Ré, La Rochelle, Communauté de communes de l'île de Ré/Université de La Rochelle, 115 p.

Le Berre S., 2008. Les observatoires de la fréquentation: outils de gestion des îles et des littoraux, thèse de doctorat en Géographie, Université de Bretagne Occidentale, 419 p.

Lussault M., 2003. Urbanité, dans LÉvy J., Lussault M. (dir.), Dictionnaire de la géographie et de l'espace et des sociétés, Paris, Belin, p. 966-967.

Pottier P., Chadenas C., Pouillaude A., Strulliou J.-F., 2009, Évaluer la capacité d'accueil et de développement des territoires littoraux, DREAL Pays de La Loire, Nantes 92 p.

Sacareau I., Vacher L., Vye D., 2010. Attractivité touristique et attractivité résidentielle du littoral charentais : lorsque les Anglais brouillent la donne, Sud-Ouest Européen, n 29 , p. 7-22.

Sacareau I., Vacher L., Vye D., 2012, La résidence secondaire est-elle un objet aux confins du tourisme? Réflexions à partir de l'exemple des résidences secondaires des Britanniques en Poitou-Charentes, Actes du Colloque international Fins et confins du tourisme, mai 2009, L'Harmattan (à paraître).

Santamaria F., 2000. La notion de « ville moyenne » en France, en Espagne et au Royaume-Uni, Annales de Géographie, vol. $109, n^{\circ} 613$, p. 227-239.

Sтоск M., 2001. Brighton \& Hove, station touristique ou ville touristique?, Revue Géographique de Lyon/Géocarrefour, vol. 76, n², p. 127-131.

Talandier M., 2007. Un nouveau modèle de développement hors métropolisation : le cas du monde rural français, thèse de doctorat en urbanisme, aménagement et politiques urbaines, $479 \mathrm{p}$.

TAulelle F., 2010. «La France des villes petites et moyennes », in Cailly L., Vanier M., La France, une géographie urbaine, Paris, Armand Colin, coll. «U », p. 149-168.

Terrier C. (dir.), 2006. Mobilité touristique et population présente - les bases de l'économie présentielle des départements, Paris, Direction du Tourisme, 128 p.

Terrier C., 2009. Distinguer la population présente de la population résidente, Courrier des statistiques, n 128, p. 63-70.

VYE D., 2005, Une lecture géographique de l'attractivité : le cas des principales villes du Centre-Ouest atlantique, thèse de doctorat en géographie, Université de Paris 13, 678 p. 